\title{
Assessment of different formation scenarios for the ring system of (10199) Chariklo
}

\author{
M. D. Melita ${ }^{1,2}$, R. Duffard ${ }^{3}$, J. L. Ortiz ${ }^{4}$, and A. Campo-Bagatin ${ }^{4,5}$ \\ ${ }^{1}$ Instituto de Astronomía y Física del Espacio (CONICET-UBA), CABA, 1428 Buenos Aires, Argentina \\ e-mail: melita@iafe.uba.ar \\ 2 Facultad de Ciencias Astronómicas y Geofísicas, Universidad Nacional de La Plata, 1900 FWA La Plata, Argentina \\ 3 Instituto de Astrofísica de Andalucía, IAA-CSIC, 18008 Granada, Spain \\ ${ }^{4}$ Departamento de Física, Ingeniería de Sistemas y Teoría de la Señal, Escuela Politécnica Superior, Universidad de Alicante, \\ 03690 Sant Vicent del Raspeig (Alacant), Spain \\ 5 Instituto de Física Aplicada a las Ciencias y la Tecnología, Universidad de Alicante, 03080 Alicante, Spain
}

Received 6 October 2016 / Accepted 8 February 2017

\begin{abstract}
Context. The discovery that the Centaur (10199) Chariklo possesses a ring system opens questions about their origin.

Aims. We here asses the plausibility of different scenarios for the origin of the observed ring system.

Methods. We first consider the possibility that the material of the ring originated in the disruption of a satellite that had reached a critical distance from the Centaur. We discuss the conditions for the putative satellite to approach the Centaur as a consequence of tidal interaction. A three-body encounter is also considered as a transport mechanism. In addition, we study the case in which the ring is formed by the ejecta of a cratering collision on the Centaur and we constrain the collision parameters and the size of the resulting crater of the event. Finally, we consider that the ring material originates from a catastrophic collision between a background object and a satellite located at a distance corresponding to the the current location of the ring. We compute the typical timescales for these scenarios.

Results. We estimate that in order to be tidally disrupted a satellite would have had to be larger than approximately $6.5 \mathrm{~km}$ at the location of the rings. However the tidal interaction is rather weak for objects of the size of outer solar system bodies at the ring location, therefore we considered other more effective mechanisms by which a satellite might have approached the Centaur. Collisonal scenarios are both physically plausible for the formation, but semianalytical estimations indicate that the probability of the corresponding collisions is low under current conditions.
\end{abstract}

Key words. Kuiper belt: general - minor planets, asteroids: general - minor planets, asteroids: individual: (10199) Chariklo

\section{Introduction}

The remarkable discovery of a ring system around (10199) Chariklo (Braga-Ribas et al. 2014), naturally raises a number of questions about their origin and the formation environment of small bodies in the outer solar system, since rings have been known so far to exist only around the four largest planets.

(10199) Chariklo is the largest known Centaur, similar in size to 2002 GZ32 (Duffard et al. 2014b). The Centaur orbit is between those of Saturn and Uranus, its semimajor axis is $15.8 \mathrm{AU}$ and its current eccentricity is 0.175 , with an effective radius of approximately $124 \mathrm{~km} \pm 9 \mathrm{~km}$, (Duffard et al. 2014a) and a geometric albedo of $0.035 \pm 0.011$ (Fornasier et al. 2013).

The ring system has two components of widths $3 \mathrm{~km}$ and $7 \mathrm{~km}$, separated by a gap of about $8 \mathrm{~km}$ and they are located between $391 \mathrm{~km}$ and $404 \mathrm{~km}$ from the Centaur and their estimated mass is equivalent to that of a body of $1 \mathrm{~km}$ radius with density of $1 \mathrm{~g} \mathrm{~cm}^{-3}$ (Braga-Ribas et al. 2014).

It has been concluded (Duffard et al. 2014a) that the dimming of the Centaur between 1997 and 2008 and the gradual decreasing of water ice features from the spectra are due to the changing view geometry of the rings. This implies that the rings are primarily composed of water ice. The steepness of the ocultation profile at the edges of the rings, the existence of the gap, as well as their short estimated spreading lifetime of only $10^{4} \mathrm{yr}$ strongly suggest that the rings are confined by kilometer-sized shepherd satellites (Braga-Ribas et al. 2014).

Further observations (Sicardy et al. 2014) revealed that the rings may have an azimuthal structure, that can be attributed to an eccentric shape of the rings, which implies that the orbits of the ring particles are precessing rigidly. Using a model where differential precession is compensated for by the self gravity of the ring (Chiang \& Goldreich 2000), Pan \& Wu (2016) have estimated the mass of the rings. Nevertheless, it has been put forward that shepherd satellites do play a fundamental role in keeping apsidal alignment (Papaloizou \& Melita 2005) and therefore this mass estimate may need to be revised. Furthermore, it is difficult to envisage the origin of the shepherd satellites in a formation scenario driven by cometary-like activity, as put forward by those authors.

It has also been suggested that the rings originated in the disruption of a parental Centaur during a close encounter with one of the giant planets (Hyodo et al. 2017), which should have taken place at about 2 planetary radii. This scenario can successfully explain not only the dynamical origin of the rings, but also their composition, since the material is extracted from the icerich mantle of the incoming body.

On the other hand, detailed dynamical simulations show that the Centaur ring system is stable (Araujo et al. 2016) under 
close encounters with the giant planets that (10199) Chariklo experienced in its past history, which did not occur at distances smaller that about 10 planetary radii.

In the following sections, we discuss several possible formation mechanisms that might explain the presence of the rings. We have studied the conditions to disrupt a satellite in the neighborhood of the Centaur, as well as different orbital transport mechanisms to that location. We have also considered the possibility that the rings have originated in either a cratering collision on the Centaur, or in a catastrophic collision on a satellite located where the rings are, and we have also estimated the probability of these events in the lifetime of typical Centaurs. In the last two sections we discuss our results and lay out our conclusions.

\section{Roche limit of (10199) Chariklo}

For a spherical satellite, the classical Roche limit, $d$, in which the self-gravity of the satellite is equal to the tidal force of the primary (the Centaur, in our case) is given by (see for example Murray \& Dermott 1999)

$d=R_{\mathrm{s}}\left(3 \frac{M}{m}\right)^{1 / 3}$,

where $R_{\mathrm{S}}$ is the radius of the satellite, $M$ is the mass of the Centaur and $m$ the mass of the satellite.

It is interesting to compare the value of the Roche limit $d$ with the so called Roche zone or Hill's radius, $d_{\mathrm{H}}$, which is the distance to the corresponding $L_{1}$ and $L_{2}$ Lagrange equilibrium points in the restricted three-body problem,

$d_{\mathrm{H}}=a\left(\frac{M}{3 M_{\odot}}\right)^{1 / 3}$,

where $a$ is the semimajor axis of the orbit of the satellite. In the case of (10199) Chariklo is approximately $d_{\mathrm{H}} \approx 2.5 \times 10^{5} \mathrm{~km}$.

We note that the shape of (10199) Chariklo seems to be very close to that of an ellipsoid of revolution with semiaxes $A=B=$ $117 \mathrm{~km}$ and $C=122 \mathrm{~km}$ (Braga-Ribas et al. 2014; Duffard et al. 2014a). Therefore its mass can be approximated as:

$M=\frac{4 \pi}{3} \rho A^{2} C$,

where $\rho$ is the unknown density of Chariklo. If we assume that the satellite is also a symmetric ellipsoid of revolution with axes $A_{\mathrm{s}}=B_{\mathrm{s}}$ and $C_{\mathrm{s}}$, its mass, $m$, can be written as:

$m=\rho_{\mathrm{s}} A_{\mathrm{s}}^{3} \epsilon$

where $\rho_{\mathrm{s}}$ is the density of the satellite and $\epsilon$ is defined as:

$\epsilon=\frac{4 \pi}{3} \gamma_{\mathrm{s}}$,

where the sphericity factor, $\gamma_{\mathrm{s}}$, is:

$\gamma_{\mathrm{s}}=\frac{C_{\mathrm{s}}}{A_{\mathrm{s}}}$.

Values of $\gamma_{\mathrm{s}}$ between 0.1 and 1.0 can be considered realistic, as these values correspond to lightcurve amplitudes of 0.4 mag's, which is the largest value observed for fast-rotator asteroids (Warner et al. 2009).

Hence, to account for the non spherical shape of both bodies, we write:

$d=\left(3 \frac{\delta}{\gamma_{\mathrm{s}}} A^{2} C\right)^{1 / 3}$,

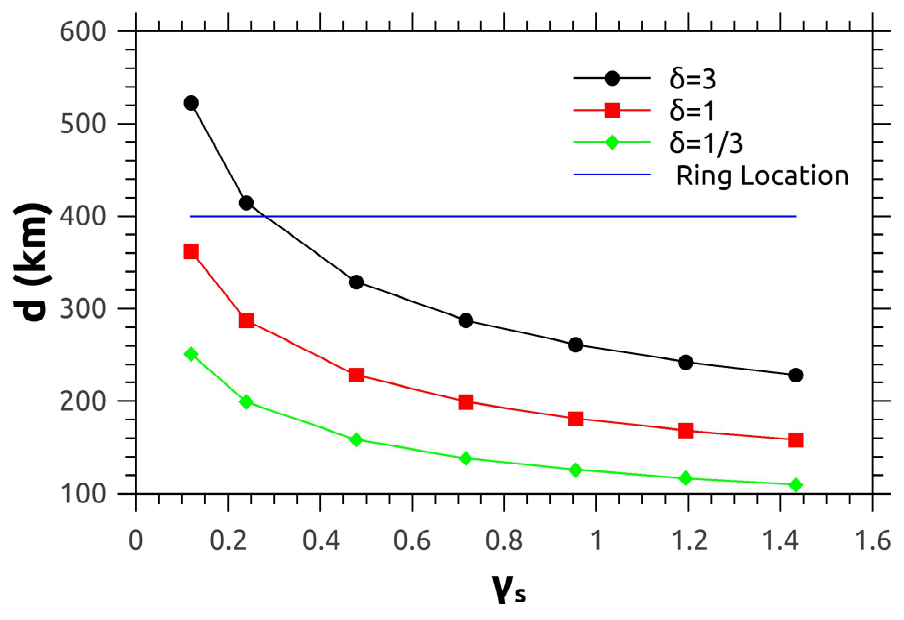

Fig. 1. Location of the Classical Roche limit, $d$, for (10199) Chariklo as a primary as a function of $\gamma_{\mathrm{s}}$ for various values of $\delta$ (see text for details).

Table 1. Disruption distance, $\Delta$, according to Dobrovolskis (1990), for not tidally locked icy bodies.

\begin{tabular}{lc}
\hline \hline Regime & Delta \\
\hline Weak & $1.37 R\left(\frac{\rho}{\rho_{\mathrm{s}}}\right)^{1 / 3}\left(\frac{p_{0}}{S}\right)^{1 / 3}$ \\
Strong & $1.19 R\left(\frac{\rho}{\rho_{\mathrm{s}}}\right)^{1 / 3}\left(1+\frac{T}{p_{0}}\right)^{-1 / 3}$ \\
\hline
\end{tabular}

where $\delta=\rho / \rho_{\mathrm{s}}$.

In Fig. 1 we plot the values of $d$ (the Roche limit distance), for (10199) Chariklo as a primary, as a function of $\gamma_{\mathrm{s}}$ for various values of $\delta$. Note that the values of $d$ are asymptotic for values of $\gamma_{\mathrm{s}}$ larger than $\approx 1.6$. For $d$ to be in the range where the rings are observed today, both those parameters take remarkably extreme values.

\subsection{Disruption distance}

Now we shall also consider the fact that the satellite material most probably possesses a non negligible tensile and/or shear strength, in order to estimate the size that an object can posses without being tidally disrupted. We work out the splitting distance, $\Delta$, depending on the strength regime and its rotational state (Dobrovolskis 1990). We consider a spherical satellite with radius $R_{\mathrm{s}}$ and density $\rho_{\mathrm{s}}$ orbiting (10199) Chariklo, with mean radius $R$ and density $\rho$. Depending on the pressure at the center of the body, given by

$p_{0}=2 / 3 \pi G \rho_{\mathrm{s}}^{2} R_{\mathrm{s}}$,

the Weak regime occurs when the tensile strength, $T$, is in the regime $T<p_{0}$, and the Strong regime when $T>p_{0}$. We assume a value of the tensile strength, $T=0.88 \rho_{\mathrm{s}}$ Davidsson (1999; density must be in I.S units, to get $T$ in Pa). We follow Dobrovolskis (1990) and, since we are interested in the low limits of distance $d$, we assume that the body is not tidally locked. In that case the disruption distance for icy materials is given in Table 1, where we have assumed that the shear-strength of ice, $S$ follows the approximate expression: $S=3 T$ (Dobrovolskis 1990).

Figure 2 shows the values of the splitting distance, $\Delta$, for the two different strength regimes described above, as a function of the satellite radius, in a case in which the satellite and the Centaur densities are both equal to $1 \mathrm{~g} \mathrm{~cm}^{-3}$, where we note that 


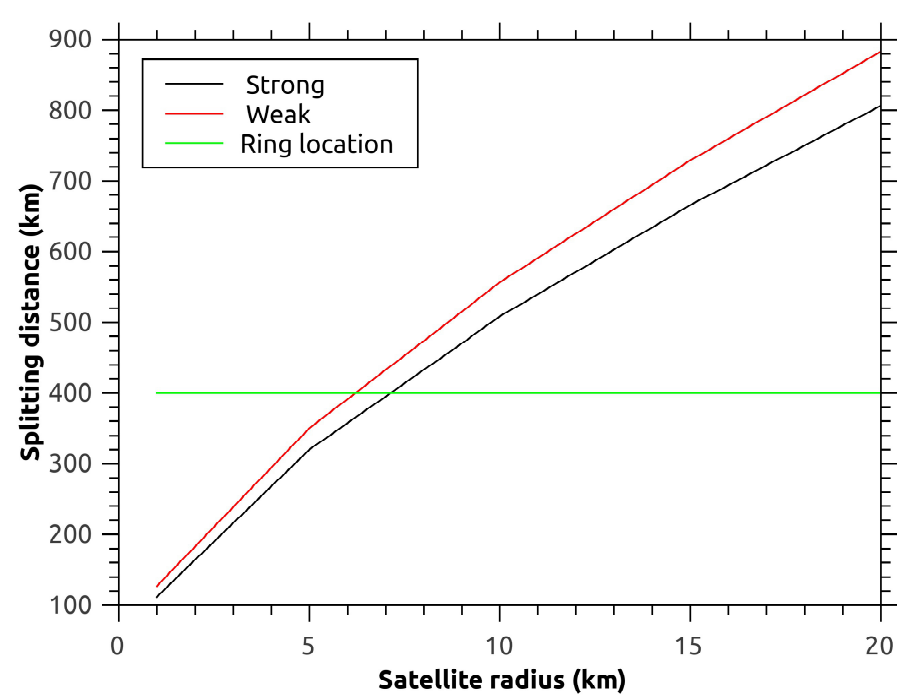

Fig. 2. Splitting distance from (10199) Chariklo as a function of the satellite radius for two different strength regimes under the assumptions of Dobrovolskis (1990).

the radius of an object has to be larger than about $6.5 \mathrm{~km}$ to be broken up at the location of the rings of (10199) Chariklo.

The validity of this result can be checked against the results of Holsapple \& Michel (2008) who computed limiting distances considering material strength, parameterized at different friction angles as well as tidal and centrifugal forces. We deduce from Fig. 4 in that article, that if the disageggated satellite is $R_{\mathrm{s}} \approx 3 \mathrm{~km}$ and is tidally locked, i.e. its intrinsic rotation period is equal to its orbital period, then the limiting distance, $d^{\prime}$, is independent of the friction angle and is given by:

$$
\frac{d^{\prime}}{R_{\mathrm{CH}}} \times\left(\frac{\rho_{\mathrm{s}}}{\rho}\right)^{1 / 3} \approx 1,
$$

where $R_{\mathrm{CH}}$ and $\rho$ are the mean radius and the density of the of the Centaur. For a satellite with no intrinsic rotation, this condition occurs for a physical radius of $R_{\mathrm{s}} \approx 2 \mathrm{~km}$, i.e. for this satellite's size the splitting distance is located at one Centaur's radii when both densities are equal (Fig. 6, Holsapple \& Michel 2008). For $d^{\prime}$ to be located at the current location of the rings, the ratio of the densities should take an unrealistic value of,

$\frac{\rho_{\mathrm{s}}}{\rho} \approx \frac{1}{40}$

On the other hand, we also deduce that an object with a similar density to that of the Centaur would have to have a radii in the range $4 \mathrm{~km}<R_{\mathrm{S}}<7 \mathrm{~km}$ and low-friction angle to disagreggate at $d^{\prime} \approx 3.6 \times R_{\mathrm{CH}}$, where the rings are located at present (Figs. 4 and 6, Holsapple \& Michel 2008), which confirms the result obtained with the model of Dobrovolskis (1990).

\section{Tidal effects}

In this section we discuss the orbital decay that is due to the tidal interaction produced by a body of the size of (10199) Chariklo on a putative satellite.

We apply the Darwin-MacDonald approximation for the tidal potential, which only considers the terms with the lowest degree on $\cos \psi$ and $R_{\mathrm{s}} / r$, where $\psi$ is the angle formed by the position vector, $\boldsymbol{r}$, and the $x-y$ plane. We also assume the classical expression of lag-angle subtended at the center of mass of

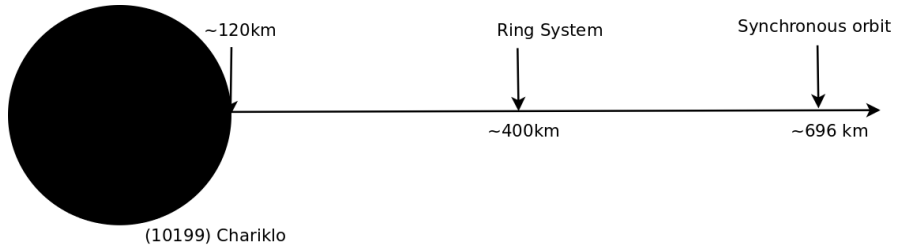

Fig. 3. Relative location of the synchronous orbit and the location of the rings.

the Centaur between the direction of its tide-raised bulge and the longitude of the satellite, $l$, that is $\sin (2 l)=1 / Q$, where $Q$ is the quality factor of the Centaur. Hence, the rate of change of the semimajor axis of the satellite that is due to the tides raised by the Centaur, can be estimated following Murray \& Dermott (1999)

$\dot{a} \approx \operatorname{sign}\left(\omega_{\mathrm{CH}}-n\right) \frac{3 k_{2}}{2 \alpha Q_{\mathrm{CH}}} a \cdot n \frac{m}{M}\left(\frac{R_{\mathrm{CH}}}{a}\right)^{5}$.

Here, we assume that the spin of (10199) Chariklo and the satellite have the same sense; $a$ is the semimajor axis of the orbit of the satellite, $n=\sqrt{G M / a^{3}}$ is the local orbital frequency in the satellite-Centaur two-body problem, $R_{\mathrm{CH}}$ is the mean radius of the Centaur, $\omega_{\mathrm{CH}}$ its intrinsic rotation frequency, $Q_{\mathrm{CH}}$ its quality factor and $k_{2}$ its Love number. We estimate the value of $k_{2}$ as (Murray \& Dermott 1999):

$k_{2}=\frac{1.5}{(1+\bar{\mu})}$.

The effective rigidity $\bar{\mu}$ of the object is estimated as (Murray \& Dermott 1999):

$\bar{\mu}=\frac{19 \mu}{[2 \rho g(A) A]}$,

where $\mu$ is the mean rigidity of the material and $g(A)$ the acceleration of gravity on its surface (Murray \& Dermott 1999). We choose $\mu=4 \mathrm{~N} \mathrm{~m}^{-2}$, which is typical of an icy body, and we obtain $k_{2}=1.6 \times 10-4$, for $\rho=1 \mathrm{~g} \mathrm{~cm}^{-3}$. We assume a typical value for icy solar system minor bodies of $Q_{\mathrm{CH}}=100$ (Table 4.1 Murray \& Dermott 1999).

From Eq. (4), we estimate the time of travel $\tau_{12}$ between two semimajor axes $a_{2}$ and $a_{1}$, that is due to the tides raised on the Centaur, as

$\tau_{12}=\operatorname{sign}\left(\omega_{\mathrm{CH}}-n\right) \frac{2}{13} C_{T}\left(a_{2}^{13 / 2}-a_{1}^{13 / 2}\right)$,

where

$C_{T}=\frac{1}{3}\left(\frac{Q}{k_{2}}\right)\left(\frac{M}{m}\right)\left(\sqrt{\frac{1}{G M}} \frac{1}{R_{\mathrm{CH}}^{5}}\right)$.

For orbital decay to occur, the satellite must be inside a synchronous orbit. On one hand, using the quoted parameters of the Centaur, the orbital period of the ring particles of (10199) Chariklo is approximately $20 \mathrm{~h}$ (see Fig. 3), which means that, for the orbit to evolve inward the rotational period of (10199) Chariklo should be - or should have been at the time of the formation of the rings - longer than that value. On the other hand, a shorter intrinsic rotational period of about $7 \mathrm{~h}$, corresponding to a double-picked light curve, has been determined for the Centaur by Fornasier et al. (2013), using SOAR (4 m) data from five non-consecutive nights. The synchronous orbit 


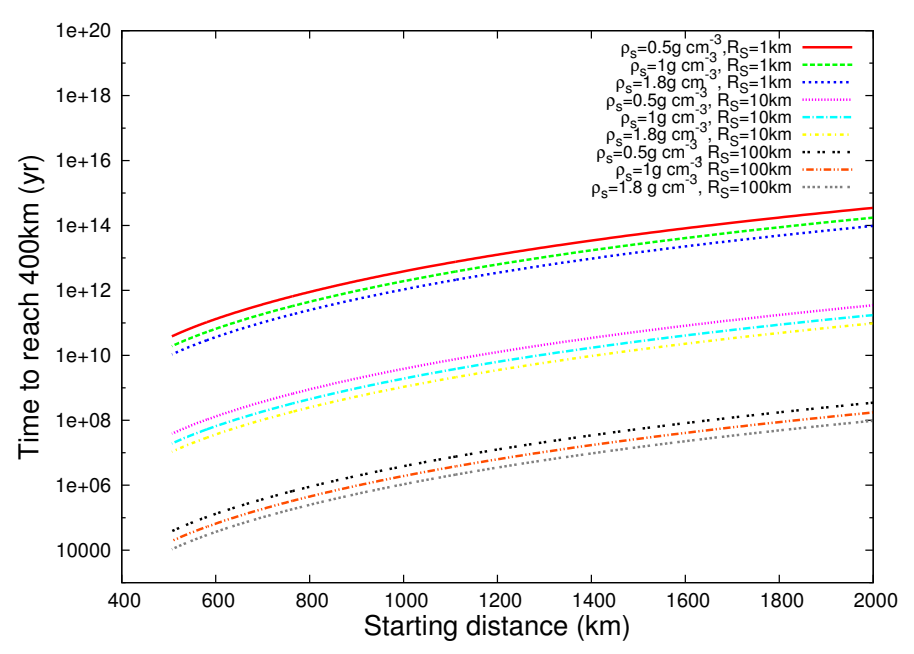

Fig. 4. Tidal-evolution travel timescale, $\tau_{12}$, due to the tides raised on the Centaur, as a function of the starting distance assumed to be larger than $500 \mathrm{~km}$, for different values of the radius of the satellite and its density.

would then be located at about $676 \mathrm{~km}$ from the Centaur, i.e. beyond the location of the rings. Therefore, the orbital evolution of a hypothetical satellite located where the rings are observed at present, would tend to expand its orbit.

We nevertheless consider the case in which the rotation rate of the Centaur may have been slower when the tidal interaction with the satellite started. Taking the final distance, $a_{2}$, as the mean location where the rings are observed today, we compute the timescale necessary to reach this distance as a consequence of the semimajor axis decay due to the tidal interaction with the Centaur. In Fig. 4 we plot this timescale as a function of the starting distance (which we assume to be larger than $500 \mathrm{~km}$ ) for different values of the radius of the satellite and its density. This timescale is shorter than the age of the solar system if the radius of the satellite is larger than $10 \mathrm{~km}$. For the semimajor axis change to have a considerable evolution in the typical dynamical lifetime of a Centaur, we find that the satellite would have had to be larger than $200 \mathrm{~km}$.

We also consider the orbital evolution caused by the tides raised by the Centaur on a hypothetical satellite. When we applying the same approximation as above, the semimajor axis change is estimated as

$\dot{a} \approx \operatorname{sign}\left(\omega_{S}-n\right) \frac{3 k_{2}(S)}{2 \alpha Q_{S}} a \cdot n \frac{M}{m}\left(\frac{R_{S}}{a}\right)^{5}$,

where $\omega_{S}$ is the intrinsic rotation rate of the satellite, $R_{S}$ its physical radius, $Q_{S}=Q_{\mathrm{CH}}=100$ its quality factor and $k_{2}(S)$, its Love number, which is estimated in a similar way as previously described for $k_{2}$, where satellite parameters are used. Now, we estimate the time of travel, $\tau_{12}^{\prime}$ between two semimajor axes locations $a_{2}$ and $a_{1}$, due to the tides raised on the satellite as:

$\tau_{12}^{\prime}=\operatorname{sign}\left(\omega_{S}-n\right) \frac{2}{13} C_{T}^{\prime}\left(a_{2}^{13 / 2}-a_{1}^{13 / 2}\right)$,

where

$C_{T}^{\prime}=\frac{2}{9}\left[\frac{Q_{S}}{k_{2}(S)}\right]\left(\frac{m}{M}\right)\left(\sqrt{\frac{1}{G M}} \frac{1}{R_{S}^{5}}\right)$.

In Fig. 5 we plot $\tau_{12}^{\prime}$ as a function of the initial orbital semimajor axis for different sizes of the satellite. We assume that $\omega_{S}<n$

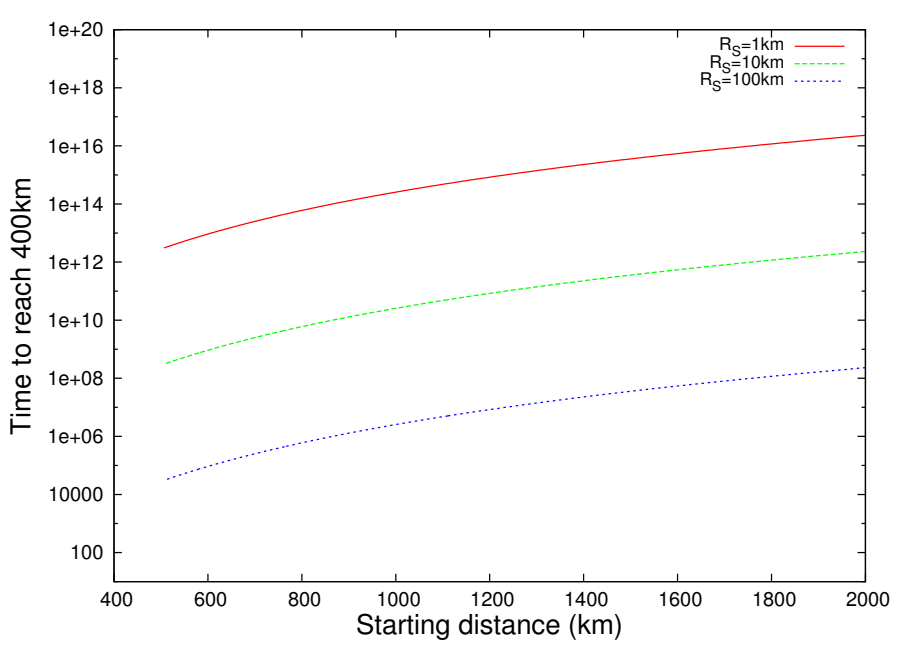

Fig. 5. Tidal-evolution travel timescale, $\tau_{12}^{\prime}$, due to the tides raised on the satellite, as a function of the starting distance assumed to be larger than $500 \mathrm{~km}$, for different values of the radius of the satellite. We also assume that the density of the satellite is $1 \mathrm{~g} \mathrm{~cm}^{-3}$.

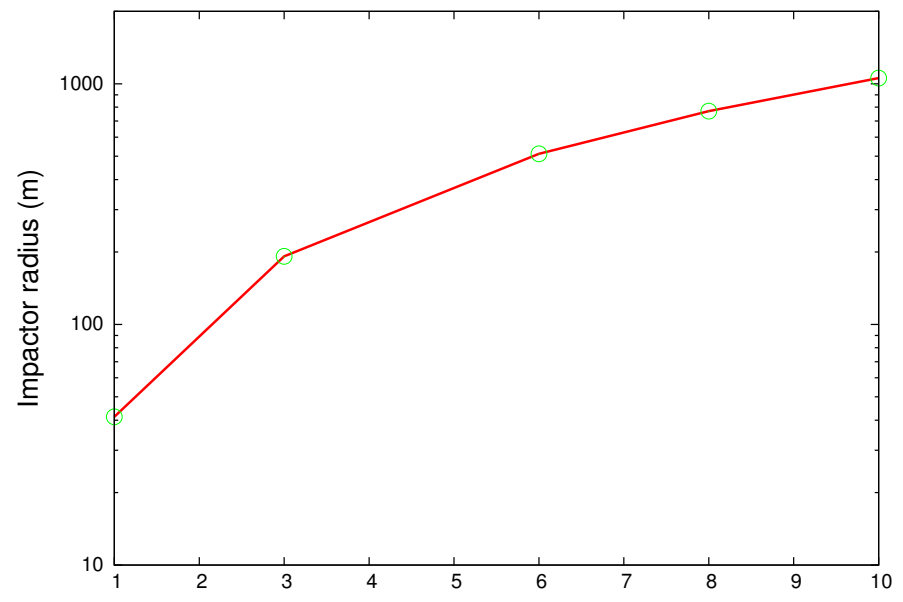

Fig. 6. Radius of the impactor that produces a shattering collision at a relative velocity of $3 \mathrm{~km} \mathrm{~s}^{-1}$, as a function of target radius. We assume that the density of both the impactor and the target satellite is $1 \mathrm{~g} \mathrm{~cm}^{-3}$.

such that the orbital evolution occurs inward. The satellite density is assumed to be equal to $1 \mathrm{~g} \mathrm{~cm}^{-3}$, but we note that plausible changes of this parameter do not alter the conclusion substantially. The values of $\tau_{12}$ are about one order of magnitude higher than $\tau_{12}^{\prime}$ and on the order of the lifetime of a Centaur if its physical radius is larger than $100 \mathrm{~km}$. Therefore we conclude that the tidal interaction is effective in bringing a satellite to a distance to the Centaur where it can be disrupted, only if the satellite size is similar to that of the Centaur itself and it is spinning more slowly.

\section{Three-body encounter}

Tidal interaction is not the only mechanism capable of bringing a body within the breakup distance to (10199) Chariklo. A threebody encounter between a Centaur and two field objects could be the cause for one or both of them to reduce its relative energy with respect to the Centaur and become gravitationally bound. The bound object can become the origin of the rings if its captured orbit occurs at a distance where tidal forces can disagreggate it. To evaluate the plausibility of this mechanism, here we shall consider a very simple model for the three-body encounter 

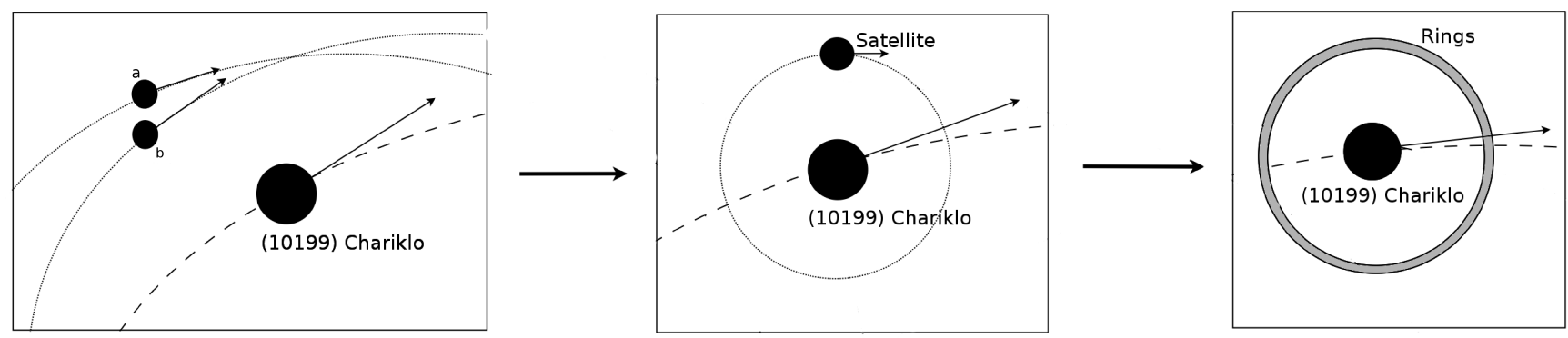

Fig. 7. Three body encounter sketch. A perfectly inelastic collision between field objects $a$ and $b$ dissipate enough energy such that the combined object end up in orbit around the Centaur.

between a Centaur of mass, $M_{\mathrm{CH}}$, and two field objects, $a$ and $b$ see Fig. 7, with masses $m_{a}$ and $m_{\mathrm{b}}$ and radii, $R_{a}$ and $R_{\mathrm{b}}$, all assumed to have spherical shape and $1 \mathrm{~g} \mathrm{~cm}^{-3}$ density, that suffer a completelly inellastic collision resulting in a single merged body.

Neglecting the mutual gravitational energy between bodies $a$ and $b$, the energy before the collision, $E_{1}$, is given by:

$$
\begin{aligned}
E_{1}= & \frac{1}{2} m_{a} V_{a}^{2}+\frac{1}{2} m_{\mathrm{b}}\left(V_{a}+\Delta V_{\mathrm{b}}\right)^{2}-\frac{G M_{\odot} m_{a}}{r_{a}}-\frac{G M_{\odot} m_{\mathrm{b}}}{r_{\mathrm{b}}} \\
& -\frac{G M_{\mathrm{CH}} m_{\mathrm{a}}}{r_{\mathrm{CH} a}}-\frac{G M_{\mathrm{CH}} m_{\mathrm{b}}}{r_{\mathrm{CHb}}}
\end{aligned}
$$

where $r_{a}$ and $r_{\mathrm{b}}$ are the heliocentric distances of bodies $a$ and $b, r_{\mathrm{CH} a}$ and $r_{\mathrm{CH} b}$ and their distances with respect to the Centaur. While the energy after the collision, $E_{2}$, is,

$E_{2}=\frac{1}{2}\left(m_{a}+m_{\mathrm{b}}\right) \cdot V_{f}^{2}-\frac{G M_{\odot}\left(m_{a}+m_{\mathrm{b}}\right)}{r}-\frac{G M_{\mathrm{CH}}\left(m_{\mathrm{a}}+m_{\mathrm{b}}\right)}{r_{\mathrm{CH}}}$

and the conservation of the linear momentum, gives:

$V_{f}=V_{a}+\frac{m_{\mathrm{b}}}{m_{a}+m_{\mathrm{b}}} \Delta V_{\mathrm{b}}$

The energy dissipated in the collision is $\Delta E=E_{2}-E_{1}$.

The gravitational energy changes with respect to the Sun and the Centaur during the collision are negligible because the distances are practically unchanged. Therefore we can write:

$\Delta E=\frac{1}{2} \frac{m_{\mathrm{b}}^{2}}{m_{a}+m_{\mathrm{b}}} \Delta V_{\mathrm{b}}^{2}$

The final orbit of the merged body is bound to the Centaur at a final distance, $a_{\mathrm{f}}$, therefore we can write,

$\frac{G M_{\mathrm{CH}}}{2 a_{\mathrm{f}}}\left(m_{a}+m_{\mathrm{b}}\right)^{2}=\frac{1}{2} \Delta V_{\mathrm{b}}^{2} m_{\mathrm{b}}^{2}$.

If $a_{\mathrm{f}}=a_{\mathrm{RINGS}}$, the radii of the bodies taking part into the collision are related by:

$R_{a}^{3}=R_{\mathrm{b}}^{3} \sqrt{\left(\frac{a_{\mathrm{RINGS}} \Delta V_{\mathrm{b}}^{2}}{G M_{\mathrm{CH}}}-1\right)}$.

We find that, for this set of assumptions, the transport is possible when the largest body has a radius of $R_{a}=6.5 \mathrm{~km}$ and it is hit at a relative velocity of $3 \mathrm{~km} \mathrm{~s}^{-1}$ by a smaller body of radius $R_{\mathrm{b}}=330 \mathrm{~m}$. Given its size, the largest body would not disrupt, as we estimated in Sect. 2.1.

\section{Collision with the nucleus of (10199) Chariklo}

Our goal is to evaluate the possibility that the mass observed in the rings of (10199) Chariklo, originated as ejecta from a collision on the surface of the Centaur. We use the scaling expression from Housen \& Holsapple (2011) to estimate the velocity, $v_{\mathrm{e}}$, and the mass, $m_{\mathrm{e}}\left(v>v_{\mathrm{e}}\right)$, of the material ejected with velocity $v>v_{\mathrm{e}}$, as a function of the distance to the center of the crater, $x$, corresponding to an impact in the gravity regime:

$m_{\mathrm{e}}\left(v>v_{\mathrm{e}}\right)=\frac{3 k}{4 \pi} \frac{\rho}{\rho_{\mathrm{s}}}\left[\left(\frac{x}{c}\right)^{3}-n_{1}^{3}\right]$

$v_{\mathrm{e}}(x)=C_{1} v_{r}\left[\frac{x}{A_{\mathrm{i}}}\left(\frac{\rho}{\rho_{\mathrm{s}}}\right)^{v}\right]^{-1 / \mu}\left(1-\frac{x}{n_{2} R}\right)^{p}$,

where $R=\left(A^{2} C\right)^{1 / 3}, v=0.4, k=0.3, p=0.3, n_{1}=1.2$, $C_{1}=0.55$, and $n_{2}=1.3$ are constants related to the icy nature of the body, and, $A_{\mathrm{i}}$ is the radius of the impactor.

We investigated which combination of distance to the center of the crater, $x$, physical radii and velocity of the impactor would produce ejecta with velocities in a range such that their final orbits would correspond to the ring system currently observed, and which amount of ejected mass, $M_{\mathrm{e}}$, is set in orbit in that location, that is,

$\frac{-G M}{2 a_{\mathrm{o}}}<\frac{-G M}{R}+\frac{1}{2} v_{\mathrm{e}}(x)^{2}<\frac{-G M}{2 a_{\mathrm{i}}}$
$M_{\mathrm{e}} \approx M_{\mathrm{RS}} \approx M\left(v>v_{\mathrm{i}}\right)-M\left(v>v_{\mathrm{o}}\right)$

where $a_{\mathrm{o}}$ and $a_{\mathrm{i}}$ are the currently observed limits of the ring system, $v_{\mathrm{o}}$ and $v_{\mathrm{i}}$, the corresponding orbital velocities, assuming circular orbits, $M\left(v>v_{\mathrm{o}}\right)$ and $M\left(v>v_{\mathrm{i}}\right)$ the mass ejected with velocities higher than the corresponding limit values of the ring system and $M_{\mathrm{RS}}$ is the order of magnitude of the mass of the ring. We also investigated how much ejected mass remains bound to the Centaur in these cases.

In Fig. 8 we plot of the combinations of impactor radii and velocity that satisfy the conditions set in Eq. (14), for each favorable pair, the corresponding values of ejected mass, $M_{\mathrm{e}}$, crater radius and mean ejection distance from it, and $\langle x\rangle$ is coded in color. The radius of the impactor is computed assuming a density of $\rho_{\mathrm{i}}=1 \mathrm{~g} \mathrm{~cm}^{-3}$ and we find that values that render favorable events are in a range between $200 \mathrm{~m}$ and $1 \mathrm{~km}$.

We also provide the corresponding plots for all the mass that remains gravitationally bound to the Centaur (right column). In all cases the mass originates at about $1 / 3$ of the diameter of the crater, the closest regions to the center of impact where the material is not compressed into the target and it is able to escape. The mass that remains in bound orbits and the mass that falls back onto the surface of the Centaur is between 2 and 3 orders 

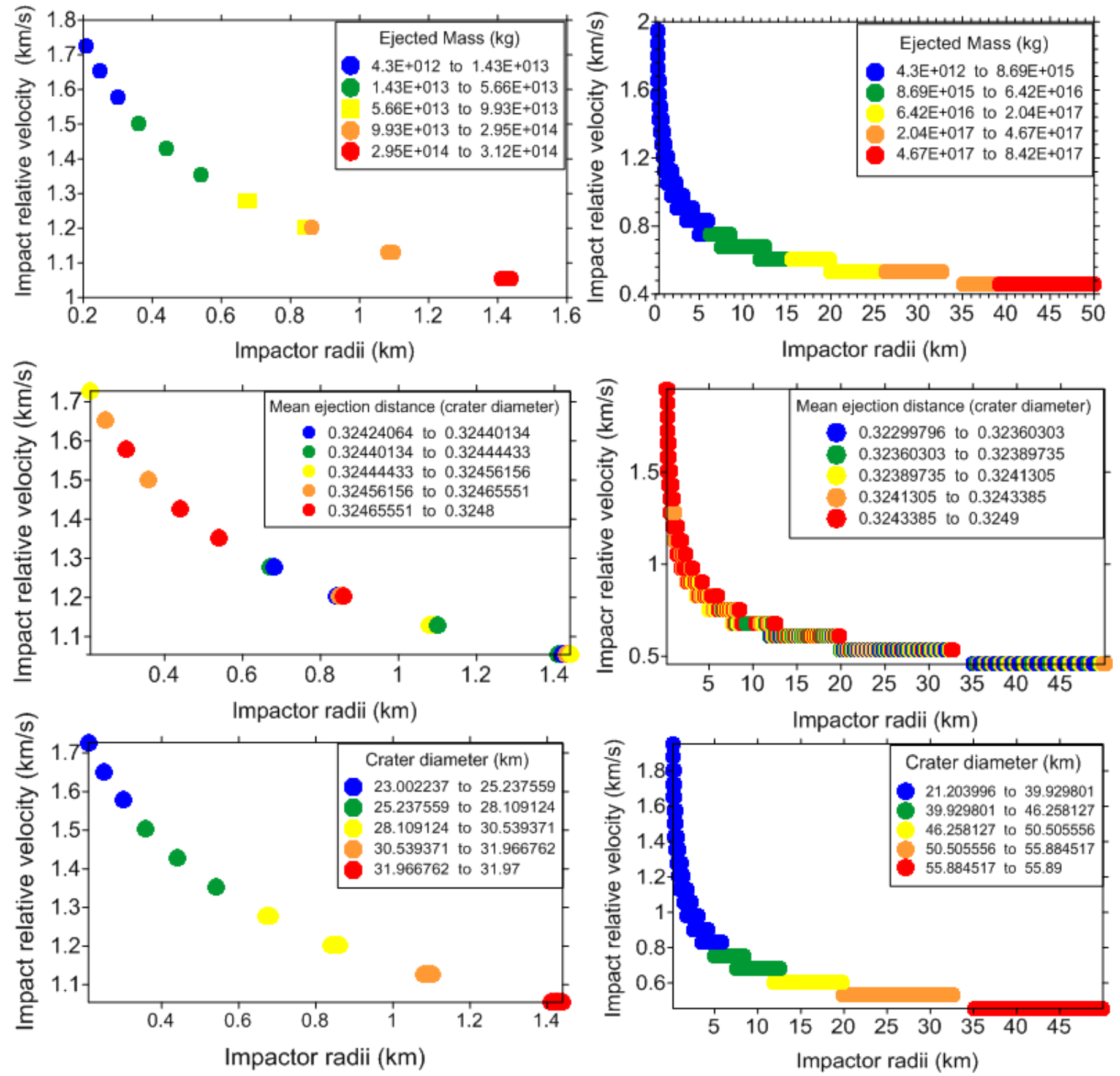

Fig. 8. Classed post plots of ejected mass, against impact relative velocity and size of the impactor. The constraint exists is that that the ejection velocities are in the range given by condition 14 .

of magnitude larger than the ejected mass that is estimated to remain in bound orbit at the currently observed location of the rings.

The past occurrence of such a collisional event could be easily corroborated, if the surface of (10199) Chariklo were observed at high resolution, because the crater in which the rings would have had originated the rings would be in $20-50 \mathrm{~km}$ in diameter.

We can calculate the probability of such an event by scaling to Chariklo the number of impacts computed by Levison et al. (2000), which give a value of $n_{\mathrm{U} \& \mathrm{~N}}=3.4 \times 10^{-4} \mathrm{yr}^{-1}$ for planets Neptune and Uranus, while we obtain $n_{\mathrm{CH}}=2.2 \times 10^{-8} \mathrm{yr}^{-1}$.
Therefore this event would be very unlikely at the current location of the body, given that the typical dynamical lifetime of a Centaur is $\sim 10^{7}$ yr (Horner et al. 2004). We note that if the collision rates in the outer solar system were an order of magnitude higher, as derived from recent observations of impacts on the giant planets (Hueso et al. 2013), the probability of this event would be marginally plausible over the dynamical lifetime of (10199) Chariklo as a Centaur. This object very likely originated in the trans-Neptunian belt and if that event were produced under current conditions, the number of required impacts per year that would be needed to produce rings as observed, $n_{\mathrm{CHTN}}$, may be estimated as

$n_{\mathrm{CHTN}} \approx \pi A^{2} P_{I} C\left(R_{1}^{-q}-R_{2}^{-q}\right)=1.3 \times 10^{-12} \mathrm{yr}^{-1}$, 
where $P_{I}=1.29 \times 10^{-22} \mathrm{~km}^{-2} \mathrm{yr}^{-1}$ is the intrinsic probability of collision of trans-Neptunian objects in present-day conditions (Dell'Oro et al. 2013), $R_{1}=0.2 \mathrm{~km}$ and $R_{2}=1.6 \mathrm{~km}$ are the minimum and maximum radii of impactors that can transfer just enough mass to the observed location, as previously determined. The size distribution of TNOs at small sizes is fairly unknown, therefore we choose a typical steadystate size distribution corresponding to $q=3,5$ (Dohnanyi 1969; O’Brien \& Greenberg 2005; Campo Bagatin \& Benavidez 2012), which should be a good approximation to the real situation unless any wavy behavior in the distribution at small sizes is present (Campo Bagatin et al. 1994), and $C=4.7 \times 10^{4}$ is a normalizing constant corresponding to the number of objects larger than $1 \mathrm{~km}$ (Petit et al. 2008).

We also note that the mean relative velocity in the transNeptunian region $v_{r}(T N)=1.65 \mathrm{~km} \mathrm{~s}^{-1}$ (Dell'Oro et al. 2013) is similar to the required velocities computed above.

Therefore, we conclude that it is highly unlikely that this type of event occurred in present-day conditions in the transNeptunian belt, during the age of the solar system.

\section{Catastrophic impact on a previously existent satellite of (10199) Chariklo}

The total mass of the ring has been estimated to be similar to that of a kilometer-sized object of density $1 \mathrm{~g} \mathrm{~cm}^{-3}$ (Braga-Ribas et al. 2014). In this section we therefore calculate the likelihood that the rings of (10199) Chariklo originated in a catastrophic impact between a field object and a satellite of the Centaur of radius $a_{\mathrm{s}}=1 \mathrm{~km}$.

According to Benz \& Asphaug (1999) the mass of the largest remnant $M_{\mathrm{LR}}$ after a catastrophic collision with (10199) Chariklo is related to the the incident kinetic energy $K_{\mathrm{i}}$ per unit mass of the target as

$\frac{M_{\mathrm{LR}}}{M_{\mathrm{Sat}}}=-s\left(\frac{K_{\mathrm{i}}}{Q^{*}}-1\right)+\frac{1}{2}$,

where $M_{\text {Sat }}$ is the mass of the Centaur's satellite, $s=0.6$ for ice for $3 \mathrm{~km} \mathrm{~s}^{-1}$ impacts. We consider an analytical dependence of $Q^{*}$ with the target radius, $R_{\mathrm{Sat}}$, as given by Benz \& Asphaug (1999), which is valid in both the gravity and the strength regimes,

$Q^{*}\left(R_{\mathrm{Sat}}\right)=Q_{0}\left(\frac{R_{\mathrm{Sat}}}{1 \mathrm{~cm}}\right)^{a}+B \rho_{\mathrm{S}}\left(\frac{R_{\mathrm{Sat}}}{1 \mathrm{~cm}}\right)^{b}$.

Constants for icy targets at $3 \mathrm{~km} \mathrm{~s}^{-1}$ are $-Q_{0}=1.0 \times 10^{7} \mathrm{erg} / \mathrm{g}$, $B=1.2 \mathrm{erg} \mathrm{cm}^{3} / \mathrm{g}, a=-0.39$ and $b=1.26$ and we consider only the case of a satellite density $\rho_{\mathrm{s}}=1 \mathrm{~g} \mathrm{~cm}^{-3}$. In the range of sizes involved, this expression coincides with that obtained by numerical simulations of asteroid collisions, which give a value of $b=1.3$ (Leinhardt \& Stewart 2009), and yield similar results.

The mass of the impactor is taken as

$m_{\mathrm{i}}=\frac{4}{3} \pi \rho_{\mathrm{i}} A_{\mathrm{i}}^{3}$,

where $A_{\mathrm{i}}$ is its mean radius and $\rho_{\mathrm{i}}$ its bulk density. The threshold catastrophic collision is defined when $\frac{M_{\mathrm{LR}}}{M_{\mathrm{Sat}}}=\frac{1}{2}$. From this, we can estimate that the radius $A_{\mathrm{i}}$ of a projectile capable of disrupting the satellite of mass $M_{\text {Sat }}$ - in an impact at a relative velocity $v_{\mathrm{i}}=3 \mathrm{~km} \mathrm{~s}^{-1}-$ is:

$A_{\mathrm{i}}=\left(\frac{3}{2 \pi \rho_{\mathrm{i}} \gamma_{\mathrm{i}}} \frac{Q^{*}\left(R_{\mathrm{Sat}}\right) M_{\mathrm{Sat}}}{v_{\mathrm{i}}^{2}}\right)^{1 / 3}$.
In Fig. 6, we plot the radius of the impactor that produces a catastrophic collision as a function of the radius of the target, Chariklo's satellite in our case. The value of the impact velocity is approximated to be the sum of the typical orbital relative velocity and the escape velocity from the Centaur, $v_{\mathrm{i}}=$ $v_{\text {esc }}+3 \mathrm{~km} \mathrm{~s}^{-1}$.

We estimate that the radius of an impactor that will catastrophically disrupt a $3 \mathrm{~km}$ satellite is $A_{\mathrm{i}} \sim 200 \mathrm{~m}$, if the bulk density of the target and the impactor are $1 \mathrm{~g} \mathrm{~cm}^{-3}$.

Since the collisional cross section of the satellite is approximately $10^{-4}$ times that of (10199) Chariklo, the estimated number of collisions with a satellite would be

$n_{\text {Sat }} \approx 10^{-12} \mathrm{yr}^{-1}$,

making this event extremely unlikely in the region where the Centaur's orbit is at present. On the other hand, if we estimate the number of such events, $n_{\text {Sat }}$, in the present-day trans-Neptunian belt, we obtain,

$n_{\mathrm{Sat}} \approx \pi A_{\mathrm{Sat}}^{2} P_{I} C A_{\mathrm{i}}^{-q}=3.2 \times 10^{-14} \mathrm{yr}^{-1}$,

where $A_{\text {Sat }}=3 \mathrm{~km}$ is the radius of the satellite and $A_{\mathrm{i}}=200 \mathrm{~m}$, which implies that this type of collision is extremely rare in the current trans-Neptunian belt.

\section{Discussion}

In the context of the remarkable discovery of a ring system about the Centaur (10199) Chariklo, the first question that arises is whether the rings lie inside or outside the corresponding classical Roche limit. We find that locating the Roche limit at about 3.3 Centaur radii, where the rings are observed, implies rather extreme physical properties for the disrupted satellite: its density would have to be $1 / 3$ that of the primary.

We considered various realistic models for the disruption distance of an object about the Centaur that lead us to conclude that an icy body with a radius of about $6.5 \mathrm{~km}$ or larger (Holsapple \& Michel 2008; Dobrovolskis 1990), would disaggregate at the ring location. The minimum amount of mass available after the disaggregation of a satellite enough to explain: a) the rings; b) the shepherd satellites capable of confining the system; and c) an object which would clear the observed gap, corresponds to an object of approximately $4 \mathrm{~km}$ radius. Therefore a very large amount of mass would have been lost from the system. If the loose material originated after the disruption did not fell between the shepherds, it would be swept away as a result of Pointing-Robertson or viscous drag in a timescale of $10^{3} \mathrm{yr}$ to $10^{4}$ yr (Braga-Ribas et al. 2014).

Even if both collision scenarios studied in Sects. 5 and 6 are physically plausible, the estimated timescales are longer than the dynamical lifetime of Centaurs, assuming typical impact probabilities taken form the literature (see for example Levison et al. 2000).

If the presently observed rotation period of Chariklo $\sim 7 \mathrm{~h}$ is primordial, the tidal interaction would have produced the expansion of the orbit. The corotation period at the current ring location is $\sim 20 \mathrm{~h}$ and - even if the Centaur spin were slower than that, tidal evolution would be too slow to produce the approach of a small satellite to its breakup distance. By means of a very simple model, we showed that a three-body encounter can be an effective mechanism to bring a field object of radius $\sim 300 \mathrm{~m}$ to its splitting distance, where the rings are observed today. It must be noted that using Eq. (16), the collision assumed in our simple model is perfectly ineastic and results in the merger of the two bodies. 


\section{Conclusions}

We estimate that the occurrence of a cratering collision on (10199) Chariklo or a catastrophic collision on a small satellite are very rare in the lifetime of a Centaur. A remarkable change on our understanding of the trans-Neptunian population is needed such that these events become plausible. For instance, the flux of incoming objects from the trans-Neptunian region should be an order of magnitude larger than what currently estimated (e.g. Hueso et al. 2013). A ring-forming collision in the primordial more massive trans-Neptunian belt might be a possible alternative.

If the ring system originated in a tidal disruption, we find that the parent body should have been approximately $6.5 \mathrm{~km}$. The disgregation of such an object produces a considerable excess with respect to the currently estimated mass. We also note that if the disrupted body were rotating faster than about $5 \mathrm{~h}$ or its shape were considerably oblated, then the size of the satellite could have been much smaller (Davidsson 2001), which would avoid the problem of the excess of mass.

The dynamical mechanism that transported the disrupted object to that distance was most probably not tidal and it might have beeen associated with a multiple encounter, although more detailed modeling is necessary on this specific issue.

Regarding the composition of the rings, if the material originated from a collision on the body of (10199) Chariklo, then they would have came from its outer crust and would most probably be composed of water ice (Hyodo et al. 2017). as observed (Duffard et al. 2014a). If the material of the rings comes form a disrupted satellite - either tidally or collisionally, then we must conclude that this body was composed mainly of water ice. On the other hand, the ring particles are in constant physical interaction with each other, therefore it is expected that their surface are constantly rejuvenated and so, the effects of cosmic irradiation are rapidly masked.

Acknowledgements. R.D. acknowledges the support of MINECO for his Ramón y Cajal Contract. Funding from Spanish funding form grant AYA-201456637-C2-1-P is acknowledged, as is the Proyecto de Excelencia de la Junta de Andalucía, J. A. 2012-FQM1776. FEDER funds are also acknowledged.
R.D. acknowledges the support of MINECO for his Ramon Cajal Contract. Funding from Spanish funding from grant AYA-2014-56637-C2-1-P is acknowledged, as is the Proyecto de Excelencia de la Junta de Andalucía, J. A. 2012FQM1776. FEDER funds are also acknowledged. M.D.M. acknowledges the support of Min CyT ANPCyt PicT 1144/2013 and the support of the travel grant from CONICET and CSiC.

\section{References}

Araujo, R. A. N., Sfair, R., \& Winter, O. C. 2016, ApJ, 824, 80

Benz, W., \& Asphaug, E. 1999, Icarus, 142, 5

Braga-Ribas, F., Sicardy, B., Ortiz, J. L., et al. 2014, Nature, 508, 72

Campo Bagatin, A., \& Benavidez, P. G. 2012, MNRAS, 423, 1254

Campo Bagatin, A., Cellino, A., Davis, D. R., Farinella, P., \& Paolicchi, P. 1994, Planet. Space Sci., 42, 1079

Chiang, E. I., \& Goldreich, P. 2000, ApJ, 540, 1084

Davidsson, B. J. R. 1999, Icarus, 142, 525

Davidsson, B. J. R. 2001, Icarus, 149, 375

Dell'Oro, A., Campo Bagatin, A., Benavidez, P. G., \& Alemañ, R. A. 2013, A\&A, 558, A95

Dobrovolskis, A. R. 1990, Icarus, 88, 24

Dohnanyi, J. S. 1969, J. Geophys. Res., 74, 2531

Duffard, R., Pinilla-Alonso, N., Ortiz, J. L., et al. 2014a, A\&A, 568, A79

Duffard, R., Pinilla-Alonso, N., Santos-Sanz, P., et al. 2014b, A\&A, 564, A92

Fornasier, S., Lellouch, E., Müller, T., et al. 2013, A\&A, 555, A15

Holsapple, K. A., \& Michel, P. 2008, Icarus, 193, 283

Horner, J., Evans, N. W., \& Bailey, M. E. 2004, MNRAS, 354, 798

Housen, K. R., \& Holsapple, K. A. 2011, Icarus, 211, 856

Hueso, R., Pérez-Hoyos, S., Sánchez-Lavega, A., et al. 2013, A\&A, 560, A55

Hyodo, R., Charnoz, S., Ohtsuki, K., \& Genda, H. 2017, Icarus, 282, 195

Leinhardt, Z. M., \& Stewart, S. T. 2009, Icarus, 199, 542

Levison, H. F., Duncan, M. J., Zahnle, K., Holman, M., \& Dones, L. 2000, Icarus, 143,415

Murray, C. D., \& Dermott, S. F. 1999, Solar system dynamics (Cambridge, UK: Cambridge University Press)

O'Brien, D. P., \& Greenberg, R. 2005, Icarus, 178, 179

Pan, M., \& Wu, Y. 2016, ApJ, 821, 18

Papaloizou, J. C. B., \& Melita, M. D. 2005, Icarus, 175, 435

Petit, J.-M., Kavelaars, J. J., Gladman, B., \& Loredo, T. 2008, Size Distribution of Multikilometer Transneptunian Objects, eds. M. A. Barucci, H. Boehnhardt, D. P. Cruikshank, A. Morbidelli, \& R. Dotson, 71

Sicardy, B., Braga-Ribas, F., Benedetti-Rossi, G., et al. 2014, in Asteroids, Comets, Meteors, eds. K. Muinonen, A. Penttilä, M. Granvik, A. Virkki, G. Fedorets, O. Wilkman, \& T. Kohout, 492

Warner, B. D., Harris, A. W., \& Pravec, P. 2009, Icarus, 202, 134 